\title{
Early detection and management of the high-risk patient with elevated blood pressure
}

\author{
Cristina Sierra \\ Alejandro de la Sierra \\ Hypertension Unit, Department of \\ Internal Medicine, Hospital Clinic. \\ University of Barcelona, Spain
}

\begin{abstract}
Severe or important blood pressure elevations are associated with the risk of cardiovascular disease. However, a significant proportion of myocardial infarctions and strokes occur in subjects with only slight elevations or even with normal blood pressure. Both the coexistence of other cardiovascular risk factors, such as diabetes or dyslipidemia, or those recently recognized, such as elevations of C-reactive protein or abdominal obesity and metabolic syndrome, or the presence of target organ damage, such as microalbuminuria, left ventricular hypertrophy, mild renal dysfunction or increased intima-media thickness, all indicate the existence of a high cardiovascular risk in mild hypertensives or in subjects with normal or high-normal blood pressure. Unfortunately, these high-risk patients are often not recognized and thus under-treated.

The 2003 European Societies of Hypertension and Cardiology guidelines emphasize the importance of a complete risk assessment and stratification in subjects at all blood pressure categories. The search for other cardiovascular risk factors and target organ damage should be encouraged. Identification of these high-risk patients may allow an earlier indication for antihypertensive treatment and for correction of all cardiovascular risk factors. The objective would be to impair the progression or to induce the regression of silent vascular damage before a clinical event develops.
\end{abstract}

Keywords: essential hypertension, target organ damage, cardiovascular risk

\section{The concept of cardiovascular risk stratification and early detection of vascular damage}

Coronary heart disease and stroke continue to be the leading causes of death and disability among adults from developed countries. Their prevalences are strongly related to the effects of many different risk factors, including high blood pressure (BP), cigarette smoking, dyslipidemia, and diabetes. Recognition that these cardiovascular (CV) risk factors often cluster together has focused attention on the concept of total $\mathrm{CV}$ risk. Thus, managing of $\mathrm{CV}$ risk factors should be viewed as an integrated strategy of intervention aimed at correcting as many of the underlying causes of $\mathrm{CV}$ disease as possible.

As the population becomes older and overweight, such as people from developed countries, the number of subjects with hypertension continues to increase. Hypertension is the most prevalent cardiovascular risk factor all over the world. Pharmacological treatment of hypertension has been shown to prolong life and to prevent or delay the development of heart failure and nephrosclerosis and to reduce the incidence of stroke (Isles et al 1986). The lower the BP attained the better has been the prognosis of the hypertensive population. Even small reductions in BP are associated with large reductions in $\mathrm{CV}$ risk, especially in hypertensive patients with additional CV risk factors such as diabetes (Hansson et al 1998).

However, treated hypertensives have an impaired survival and an increased mortality from CV disease compared to non-hypertensive subjects of similar age 
(Andersson et al 1998). A late start of antihypertensive therapy, an inadequate control of BP and/or the type of therapy employed, or an incomplete and simultaneous coverage of the different simultaneous components of the global CV risk, could account for the worse prognosis in the hypertensive population. Recently published Guidelines (Chobanian et al 2003; European Society of Hypertension 2003) have recognized the need for a strict BP control in every hypertensive patient while stressing the existence of compelling indications for the utilisation of certain drugs in particular when diabetes mellitus or target organ damage are present. In this sense, an early detection of hypertensive vascular damage and, consequently, adequate cardiovascular risk stratification appears to be one of the most important strategies in order to prevent the development of $\mathrm{CV}$ disease (De Backer et al 2003). Indeed, the future of hypertension would aim at the treatment of the global CV risk of the patient, it means that it is necessary to look at the $\mathrm{CV}$ disease as a continuum, to consider all the CV risk factors present in a single patient and, for this, it is mandatory an earlier detection of vascular damage, considering that the earlier the treatment the better the prognosis of the patient.

Hypertension is a well established risk factor for the development of all of the clinical manifestations of atherosclerosis and cardiovascular disease. Atherosclerosis is a generalized disease of the arterial wall, which may progress or regress depending on a plethora of factors (Badimon et al 1993). This dynamic process is characterized by arterial wall remodelling that may go unnoticed for a lifetime, but may also present as acute vascular disease and become clinically manifest. An early detection of the atherosclerotic disease (before clinical manifestations have become evident) has received an intense attention in the last years. The presence of what are called surrogate markers of disease might be used to investigate determinants of atherosclerosis at an early stage of the process and can, subsequently, assess modifiers of atherosclerosis disease progression, such as lifestyle and pharmacological interventions. In the future, research should be aimed at controlling or reversing subclinical target organ damage.

\section{Cardiovascular risk stratification in hypertensive patients: what is important?}

Elevated BP has been found to be related to the development of $\mathrm{CV}$ disease in a continuous, graded fashion, with no indication of a critical value. Indeed, the risk of $\mathrm{CV}$ sequelae increases with each increment in BP, even within the normal range. In a subgroup of the Framingham Heart Study, those people with high-normal BP, defined as systolic BP of 130-139 mmHg, diastolic BP of 85-89 mmHg, or both, had higher $\mathrm{CV}$ event rates than those with optimal $\mathrm{BP}$, defined as systolic BP of less than $120 \mathrm{mmHg}$ and diastolic BP of less than $80 \mathrm{mmHg}$ (Vasan et al 2001). These findings provide further support to the theory that patients with high-normal $\mathrm{BP}$ need to be identified and managed differently from subjects with normal or optimal BP.

In this sense, and according to the European Guidelines for managing high BP, due to the presence of additional risk factors (Table 1), target organ damage (Table 2), and associated clinical conditions (Table 3), patients may be at high risk of $\mathrm{CV}$ events even when their BP is normal or high-normal (systolic BP 120-139 mmHg; diastolic BP: 80-89) (Table 4). Such high-risk patients, although common in clinical practice, are often unrecognized and under-treated. Most experts recommend an integrated approach of risk management to prevent the complications of raised BP. Accordingly, the need to start treatment increases in the presence of other $\mathrm{CV}$ risk factors or when absolute risk reaches a specified threshold. Risk scores profiles are based on Framingham Study data (Low, Moderate, High or Very High CV risk mean probabilities to develop CV disease in the next 10 years of $<15 \%, 15 \%-20 \%, 20 \%-30 \%$, and $>30 \%$ respectively), as well as SCORE data $(<4 \%, 4 \%-5 \%, 5 \%-8 \%$, and $>8 \%$ for low, moderate, high, and very high risk of fatal $\mathrm{CV}$ disease, respectively).

Recognition of the presence of hypertensive target organ damage, such as left ventricular hypertrophy (LVH), microalbuminuria (MAB) or increased carotid intima media thickness (IMT) is highly important and indicates the necessity of beginning antihypertensive therapy in these hypertensive patients. As mentioned earlier, in the vast majority

Table I Risk factors for cardiovascular disease used for stratification

Levels of systolic and diastolic blood pressure

Men $>55$ years

Women $>65$ years

Smoking

Dyslipidemia: total cholesterol $>250 \mathrm{mg} / \mathrm{dl}(6.5 \mathrm{mmol} / \mathrm{l})$, or LDLcholesterol $>155 \mathrm{mg} / \mathrm{dl}(4 \mathrm{mmol} / \mathrm{l})$, or HDL-cholesterol $<40 \mathrm{mg} / \mathrm{dl}$ $(1.0 \mathrm{mmol} / \mathrm{l})$ in men or $<48 \mathrm{mg} / \mathrm{dl}(1.2 \mathrm{mmol} / \mathrm{l})$ in women

Family history of premature cardiovascular disease $(<55$ years in men or $<65$ years in women)

Abdominal obesity: abdominal circumference $\geq 102 \mathrm{~cm}$ in men or

$\geq 88 \mathrm{~cm}$ in women

C-reactive protein $\geq 1 \mathrm{mg} / \mathrm{dl}$

From ESH/ESC Guidelines (2003). 
Table 2 Indicators of target organ damage

Left ventricular hypertrophy: Electrocardiogram (Sokolow-Lyon $>38 \mathrm{~mm}$; Cornell $>2440 \mathrm{~mm} * \mathrm{~ms}$ ). Echocardiogram (left ventricular mass index $\geq 125 \mathrm{~g} / \mathrm{m}^{2}$ in men or $\geq 110 \mathrm{~g} / \mathrm{m}^{2}$ in women. Ultrasound evidence of arterial wall thickening (carotid intima-media thickness $\geq 0.9 \mathrm{~mm}$ ) or atherosclerotic plaque. Slight increase in serum creatinine: $1.3-1.5 \mathrm{mg} / \mathrm{dl}(\mathrm{I} 15-133 \mu \mathrm{mol} / \mathrm{l})$ in men or $1.2-1.4 \mathrm{mg} / \mathrm{dl}(107-124 \mu \mathrm{mol} / \mathrm{l})$ in women. Microalbuminuria: urinary albumin excretion $30-300 \mathrm{mg} / 24 \mathrm{~h}$. Albumin-to-creatinine ratio $\geq 22 \mathrm{mg} / \mathrm{g}(2.5 \mathrm{mg} / \mathrm{mmol})$ in men or $\geq 31 \mathrm{mg} / \mathrm{g}(3.5 \mathrm{mg} / \mathrm{mmol})$ in women.

From ESH/ESC Guidelines (2003).

of hypertensive patients the occurrence of major CV events is the result of long-term exposure to multiple risk factors, and is usually preceded by the development of asymptomatic abnormalities at the vascular and cardiac level (Devereux et al 1993). This so-called silent target organ damage phase represents an intermediate end-point and, in turn, a strong independent predictor of an unfavourable outcome.

On the other hand, when a hypertensive patient has type 2 diabetes or other 3 or more $\mathrm{CV}$ risk factors also represents a high risk for $\mathrm{CV}$ disease. Optimal $\mathrm{CV}$ protection in hypertension requires take into account not only the severity of the $\mathrm{BP}$ elevation but also the often associated CV risk factors (dyslipidemia, diabetes, obesity, smoking...), or the presence of target organ damage (LVH, MAB, IMT...). Recently, two new $\mathrm{CV}$ risk factors, the elevated C-reactive protein (CRP) and the cluster of metabolic abnormalities, known as metabolic syndrome (MS) have received a great interest due to their close association with $\mathrm{CV}$ disease.

\section{What about C-reactive protein and the risk of CV disease?}

Improvements in risk stratification by identification of new biomarkers have been extensively investigated in the past decade. A substantial number of biomarkers, representing various stages of atherosclerotic process and/or impaired cardiovascular function have been evaluated in addition to traditional risk factors. There is strong evidence that inflammation plays a key role in the cascade of atherosclerosis, both in its development and in its complications due to plaque instability and rupture.

Various clinical studies have shown that elevated C-reactive protein (CRP) levels in healthy populations predict vascular events, such as myocardial infarction and stroke (Wilson et al 2006), and that measurements of serum levels of CRP using a high sensitivity assay (hsCRP) could be a powerful research tool in assessing at-risk populations.
In fact, elevated serum CRP level has been shown to be a stronger predictor of incident $\mathrm{CV}$ events in dyslipidemic men than LDL-cholesterol, and it has a predictive capacity when added to the Framingham risk score (Ridker et al 2002), Moreover, hs-CRP is also predictive in subjects with metabolic syndrome (Ridker et al 2003). Finally, in apparently healthy men and women, a significant relationship was found between elevation of BP, hsCRP and interleukin-6, and the combination of hsCRP and hypertension was additive in terms of risk prediction (Blake et al 2003).

On the other hand, there are some data suggesting that aspirin, angiotensin converting enzyme inhibitors (ACE), and HMG Co-A reductase inhibitors (statins), which all reduces vascular event rates, also reduce serum levels of hsCRP, and therefore hsCRP may potentially guide therapy.

High-sensitivity CRP is useful in those patients whose risk stratification is unclear, especially in those subjects with high normal blood pressure or grade I hypertensives with two additional established cardiovascular risk factors. Elevated hsCRP may be an indication of pharmacological therapy in these patients.

\section{Impact of metabolic syndrome on CV disease in hypertensive patients}

It has been long recognized that hypertension is often a part of a wider constellation of metabolic abnormalities that includes abdominal (or visceral) obesity, a characteristic dyslipidemia (low HDL-cholesterol and high triglycerides), glucose intolerance, and insulin resistance, which are the main features of the metabolic syndrome (MS). MS is a relatively common condition in patients with hypertension, and its frequency has been reported as being around $30 \%$, depending on the clinical characteristics and the criteria used (Cuspidi et al 2004). The presence of MS has been shown to confer an increased risk of $\mathrm{CV}$ events, which may, in part, be attributed to the individual risk factors which concur in defining it, and in part to a cluster of other, often unmeasured variables such

Table 3 Associated clinical conditions (clinically evident cardiovascular or renal disease)

Cerebrovascular disease: ischemic stroke, cerebral hemorraghe, transient ischemic attack

Heart disease: myocardial infarction, angina, coronary revascularization, congestive heart failure

Renal disease: diabetic nephropathy, renal impairment (serum creatinine $>$ $1.5 \mathrm{mg} / \mathrm{dl}$ (I $33 \mu \mathrm{mol} / \mathrm{l})$ in men or $>1.4 \mathrm{mg} / \mathrm{dl}(\mathrm{I} 24 \mu \mathrm{mol} / \mathrm{l})$ in women Peripheral vascular disease

Advanced retinopathy: hemorraghes or exudates, papilloedema

From ESH/ESC Guidelines (2003) 
Table 4 Stratification of risk and prognosis

\begin{tabular}{|c|c|c|c|c|c|}
\hline \multirow[b]{2}{*}{$\begin{array}{l}\text { Other RF and } \\
\text { disease history }\end{array}$} & \multicolumn{5}{|c|}{ Blood pressure $(\mathrm{mmHg})$} \\
\hline & $\begin{array}{l}\text { Normal } \\
\text { SBP I 20-I } 29 \text { or } \\
\text { DBP } 80-84\end{array}$ & $\begin{array}{l}\text { High-normal } \\
\text { SBP I30-139 or } \\
\text { DBP } 85-89\end{array}$ & $\begin{array}{l}\text { Grade I } \\
\text { SBP I 40-I59 or } \\
\text { DBP 90-99 }\end{array}$ & $\begin{array}{l}\text { Grade } 2 \\
\text { SBP } 160-179 \text { or } \\
\text { DBP } 100-109\end{array}$ & $\begin{array}{l}\text { Grade } 3 \\
S B P \geq 180 \text { or } \\
D B P \geq 110\end{array}$ \\
\hline No other RF & Average risk & Average risk & Low added risk & Moderate added risk & High added risk \\
\hline $\mathrm{I}-2 \mathrm{RF}$ & Low added risk & Low added risk & Moderate added risk & Moderate added risk & Very high added risk \\
\hline $\begin{array}{l}3 \text { or more RF or } \\
\text { TOD or Diabetes }\end{array}$ & Moderate added risk & High added risk & High added risk & High added risk & Very high added risk \\
\hline $\mathrm{ACC}$ & High added risk & Very high added risk & Very high added risk & Very high added risk & Very high added risk \\
\hline
\end{tabular}

Abbreviations: RF, risk factors; SBP, systolic blood pressure; DBP, diastolic blood pressure; TOD, target organ damage; ACC, associate clinical conditions. From ESH/ESC Guidelines (2003).

as a proinflammatory state, an impaired fibrinolysis and an increased oxidative stress, which usually go along with it (Grundy 1999). Recently, it has been demonstrated that the adverse prognostic impact of MS may also be extended to hypertensive patients (Schillaci et al 2004). Indeed, in a prospective observational study of Italian adult subjects with essential hypertension, those patients with this syndrome (34\% of the whole population) carried an increased risk of developing cardiac and cerebrovascular events.

Some recent studies reported an increased prevalence of LVH, diastolic dysfunction, early carotid atherosclerosis, impaired aortic distensibility, hypertensive retinopathy and MAB in hypertensive patients with MS when compared to those without it (Mule et al 2006). The increased occurrence of these signs of subclinical target organ damage, most of which are recognized as significant independent predictors of adverse $\mathrm{CV}$ and renal outcomes, may partially explain the association of the MS with a higher $\mathrm{CV}$ and renal risk.

\section{Implications of microalbuminuria in hypertensive patients}

The presence of MAB (elevated urinary albumin excretion below the proteinuric level, ie, MAB: $30-300 \mathrm{mg} / 24 \mathrm{~h}$ or urinary albumin/creatinine ratio $\geq 2.5 \mathrm{mg} / \mathrm{mmol}$ in men, and $\geq 3.5 \mathrm{mg} / \mathrm{mmol}$ in women) has been long recognized as a marker of kidney disease and increased $\mathrm{CV}$ risk in both types 1 and 2 diabetes mellitus. Further clinical evidence documented an association between $\mathrm{MAB}$ and other $\mathrm{CV}$ risk factors, target organ damage and risk of $\mathrm{CV}$ disease in the general population and in specific clinical contexts including essential hypertension. There appears to be a linear relationship between the degree of MAB and CV risk. Evaluation of MAB is one of the recommended laboratory tests proposed by the European Society of Hypertension Guidelines to assess target organ damage in hypertensive subjects. In this hypertensive population, the reported prevalence of
MAB ranges from about $4 \%$ to $46 \%$ across different studies (Reboldi et al 2005). These differences may be explained by the huge intraindividual variability in MAB, age and ethnicity, discrepancies in the technique of measurement and different definitions of MAB. Increased MAB has been associated with subclinical hypertensive organ damage. In fact, a higher prevalence of concentric LVH and subclinical impairment of LV performance, as well as the presence of carotid atherosclerosis have been all reported in patients with MAB (Pontremolli et al 2002). These associations might per se justify a greater incidence of $\mathrm{CV}$ events in hypertensive patients with MAB. In a 10-year prospective study of more than 2000 patients with hypertension, a urinary albumin/creatinine ratio $>1.07 \mathrm{mg} / \mathrm{mmol}$ strongly and independently predicted ischemic heart disease, more than doubling the risk (Jensen et al 2000).

Measurement of MAB is an inexpensive test, widely available, suitable for use in clinical practice, which could represent a useful approach for risk stratification and for identifying patients at high $\mathrm{CV}$ risk.

\section{Implications of left ventricular hypertrophy in hypertensive patients}

In 1969, the Framingham study already demonstrated that once LVH is recognized clinically by electrocardiography, it represents a strong predictor for $\mathrm{CV}$ disease. Lately, several studies have reported that electrocardiographically or echocardiographically determined LVH is an independent risk factor for cardiovascular morbidity and mortality in essential hypertension (Casale et al 1986; Koren et al 1991). Cardiovascular event rates are 2 to 4 fold higher in the presence of LVH. In hypertension, LVH is initially a useful compensatory process to abnormal loading conditions but it is also the first step toward the development of overt clinical disease, such as congestive heart failure, ischemic heart disease, cardiac dysrhythmias, and stroke. It has also 
been demonstrated that left ventricular geometric patterns add prognostic information to both the development of cardiovascular disease (Koren et al 1991) and the presence of extracardiac target organ damage in essential hypertension. Indeed, hypertensive patients with concentric LVH have more advanced target organ damage, such as renal (Shigematsu et al 1998; Pontremolli et al 1999) retinal (Pontremolli et al 2002), and silent cerebrovascular (Sierra et al 2002) involvement, than those with other patterns of left ventricular geometry.

A search for the detection of LVH in electrocardiograms must be a part of routine clinical examination to all patients with $\mathrm{CV}$ disease or at risk of CV events. The systematic procedure of an echocardiogram depends on each site facilities, but it can be especially useful in those patients with highnormal blood pressure or grades I or II hypertension without evidence of other signs of target organ damage. The presence of $\mathrm{LVH}$ in this group of patients indicates a need for a more aggressive management of $\mathrm{CV}$ risk factors in order to further prevent $\mathrm{CV}$ damage.

\section{Implications of increased intima media thickness in hypertensive patients}

B-mode ultrasound imaging technology has evolved to such an extent that the walls of superficial arteries, such as carotid or femoral arteries, can be imaged noninvasively, in real time and at high resolution. Arterial wall thickness can be measured as a continuous variable from childhood into old age. Large observational studies, such as the Rotterdam study (Bots et al 1997), and the ARIC study (ARIC Investigators 1997), and atherosclerosis regression trials of lipid and BP modifying pharmacotherapy have established that increased intima-media thickness (IMT) of the carotid and femoral arteries, as measured noninvasively by B-mode ultrasound, is a valid surrogate marker for the progression of atherosclerotic disease (Mancini et al 2004). More compelling is evidence showing that carotid atheroma is a predictor of vascular events and that is useful for risk stratification. In the ARIC study, a strong and graded relationship was observed between coronary heart disease incidence and carotid IMT. Hazard ratio comparing extreme mean IMT ( $\geq 1 \mathrm{~mm}$ ) to not extreme $(<1 \mathrm{~mm})$ yielded 5.07 for women and 1.85 for men. The incidence and relative risk of new $\mathrm{CV}$ events correlated with measurements of carotid IMT after adjustments for age, gender, and traditional risk factors.

In the European Guidelines for the management of hypertension, IMT was included as one of the recommended procedures to assess target organ damage in hypertensive subjects. In this sense, an IMT $\geq 0.9 \mathrm{~mm}$ is considered as a marker of target organ damage in essential hypertension.

\section{Treatment of hypertensive patients at risk of developing cardiovascular diseases Hypertension and microalbuminuria}

The goals of treatment are regression of MAB and prevention of the progression to persistent clinical albuminuria. Treatments that lower MAB are also associated with $\mathrm{CV}$ protection. First of all, a tight BP control has demonstrated to slow the progression of the disease compared to less tight BP control (UK Prospective Diabetes Study Group 1998; Schrier et al 2002).

Angiotensin receptor blockers (ARBs) are considered the first line treatment in patients with type 2 diabetes and nephropathy at different clinical stages. In hypertensive patients with type 2 diabetes mellitus and MAB, treatment with irbesartan $300 \mathrm{mg}$ daily significantly decreased the rate of progression to overt nephropathy (proteinuria) (Parving et al 2001). Similar results were found with valsartan compared to amlodipine (Viberti et al 2002).

Although there is no doubt of the usefulness of ARBs in the prevention and treatment of type 2 diabetic nephropathy, one important criticism of studies is that the use of ACE inhibitors was not allowed in patients enrolled in these clinical trials and there was no direct comparison between ARBs and ACE inhibitors. The recently reported DETAIL study compared telmisartan vs enalapril in 250 type 2 diabetics with microalbuminuria $(80 \%)$ or proteinuria $(20 \%)$ and normal serum creatinine (Barnett et al 2004). The primary endpoint was the change in the glomerular filtration rate. At the end of the 5-year observation period there were no differences between the two treatment regimens.

Another point of interest is the possible additive effect of $\mathrm{ARBs}$ and $\mathrm{ACE}$ inhibitors on renal protection. The COOPERATE study comparing the combination of losartan and trandolapril against monotherapy with either drug in patients with non-diabetic nephropathy showed a greater effect of the combination on a composite endpoint of doubling the serum creatinine and end-stage renal disease (Nakao et al 2003). These results suggest the possibility of using combined RAS blockade in the protection of hypertensive patients with renal disease.

MAB can also be prevented. In the BENEDICT study (Ruggenenti et al 2004), trandolapril alone or in combination with sustained release verapamil prolonged the time to the 
onset of persistent microalbuminuria compared with placebo or verapamil alone in normoalbuminuric type 2 diabetics.

\section{Hypertension and left ventricular hypertrophy}

LVH and its regression with antihypertensive treatment clearly influence the risk of future cardiovascular events (Verdecchia et al 1998). Several meta-analyses have examined the effect of the main antihypertensive drug classes on LVH regression, the most recent of which included studies with ARBs and concluded that LVH regression with ARBs was similar to that observed with ACE inhibitors or calcium channel blockers. These three classes of drugs were more potent LVH regressors than classic treatment with diuretics or betablockers (Klingbeil et al 2003). The comparison of losartan versus atenolol in the LIFE trial also showed that losartan produced greater LVH regression than atenolol (Dahlöf et al 2002). This was demonstrated by measuring ECG indexes of LVH (the Sokolow-Lyon and the Cornell product) in the whole group of patients, or by measuring left ventricular mass index by echocardiography in a selected subgroup of patients (Devereux et al 2004).

\section{Hypertension and intima media thickness}

Few studies have evaluated the effect of antihypertensive agents on IMT progression. The ELSA study was performed in 2334 hypertensive patients (aged 45-75 years) who were randomized to receive lacidipine or atenolol for 4 years, in order to compare the effects of treatments on IMT (Zanchetti et al 2002). Results showed a greater efficacy of lacidipine with respect to atenolol on carotid IMT progression and on the development of new atherosclerotic plaques.

A sub-study of the INSIGHT trial also compared the effects of long-acting nifedipine versus a diuretic combination (hydrochlorothiazide + amiloride) on IMT progression in 439 hypertensive patients aged 55-80 years and one or more additional risk factors. In the until-end-of-study population, IMT progression rates were different between groups in favour of long-acting nifedipine (Simon et al 2001).

Finally, the CAMELOT study was carried out to evaluate the effect of antihypertensive agents on CV events in patients with coronary artery disease and normal BP (defined as diastolic BP $<100 \mathrm{mmHg}$ with or without treatment). A substudy evaluated the change in percent coronary atheroma volume determined by intravascular ultrasonography in 274 patients. Amlodipine, enalapril or placebo were compared. There was a trend toward less progression of atherosclerosis in the amlodipine group versus placebo $(\mathrm{p}=0.12)$. However, in the subgroup with baseline $\mathrm{BP}$ above the mean, significant reduction in progression was observed in the amlodipine group compared with placebo $(p=0.02)$. With respect to baseline, there was progression in the placebo group ( $\mathrm{p}<0.001)$, a trend toward progression in the enalapril group $(\mathrm{p}=0.08)$, and a lack of progression in the amlodipine group $(\mathrm{p}=0.31)$.

\section{Early detection of patients at high risk: early $\mathrm{BP}$ reduction?}

The VALUE trial compared two active antihypertensive treatments (valsartan and amlodipine) in high-risk hypertensives older than 50 years (Julius et al 2004). At the end of the study there were no differences in the primary endpoint, which occurred in $10.6 \%$ of valsartan-treated patients and in $10.4 \%$ of amlodipine-treated patients. Some of the prespecified secondary endpoints were favourable to amlodipine with respect to valsartan, including myocardial infarction and stroke, whereas valsartan slightly reduced the development of heart failure.

The VALUE trial showed important differences in the BP reduction achieved by the two treatment regimens. BP differences were especially apparent during the first part of the study (4/2.1 mmHg in the first month) and maintained at more than $1 \mathrm{mmHg}$ during all the follow-up in favor of amlodipine. In order to separate the BP dependent and independent effects of antihypertensive treatment, the VALUE investigators carried out a special case-control analysis choosing more than 5000 pairs of patients matched for age, sex, risk and, especially, for systolic BP (Weber et al 2004). Using this approach, differences in the cardiovascular endpoint that favoured amlodipine in the main analysis disappeared. However, the main conclusion of the VALUE trial was that early BP reduction was clearly more important than the type of treatment used for the prevention of cardiovascular disease, at least in high-risk hypertensives.

\section{Newer or classical antihypertensive agents?}

The issue of which antihypertensive agent should be used in first-line treatment has been controversial for almost two decades. However, to reach the target of BP recommended in international guidelines, two or more antihypertensive agents need to be used in most patients. On the other hand, the question if there are differences between the newer antihypertensive agents, such as calcium channel blockers, ACE inhibitors, and ARBs compared with classical agents, such as diuretics and beta-blockers, remains controversial. It has 
been recently published the ASCOT-BPLA study (Dahlöf et al 2005) which tried to answer this question. This study included 19257 hypertensive patients aged 40-79 years with at least three other CV risk factors. Patients were randomised to receive amlodipine, adding perindopril as required, or atenolol, adding bendrofluthiazide as required. By the end of the trial, only $15 \%$ and $9 \%$ were taking amlodipine and atenolol monotherapy, respectively. Interpretation of the results show that amlodipine-based regimen prevented more major CV events and induced less diabetes than the atenololbased regimen, and that these effects might not be entirely explained by better control of BP.

On the other hand, the new-onset diabetes that can occur with some antihypertensive drugs need to be further investigated. However, there are some evidences supporting the idea that calcium channel blockers, ACE inhibitors, and ARBs offers a better metabolic profile than classical agents. These data are particularly important in the treatment of high BP in the metabolic syndrome.

\section{Closing remarks}

In addition to patients with severe elevation of BP or those with previous $\mathrm{CV}$ disease, it has become more evident that slight elevations of $\mathrm{BP}$, even within the normal range might be accompanied by other $\mathrm{CV}$ risk factors and especially by silent target organ damage, such as microalbuminuria, LVH or increased IMT, that are only evident by specific search.

Identification of these high-risk patients should be encouraged, due to the fact that strict BP control and the use of certain types of antihypertensive therapy may help to effectively reduce CV mortality and morbidity.

\section{References}

Anderson OK, Almgren T, Persson B, et al. 1998. Survival in treated hypertension: follow-up study after two decades. BMJ, 317:167-71.

ARIC Investigators. 1997. Association of coronary heart disease incidence with carotid arterial wall thickness and major risk factors: the Atherosclerosis Risk in the Communities (ARIC) study. Am J Epidemiol, 146:483-94.

Badimon JJ, Fuster V, Chesebro JH, et al. 1993. Coronary atherosclerosis: a multifactorial disease. Circulation, 87 (Suppl):II3-16.

Barnett AH, Bain SC, Bouter P, et al. 2004. Angiotensin-receptor blockade versus converting-enzyme inhibition in type 2 diabetes and nephropathy. N Engl J Med, 351:1952-61.

Blake GJ, Rifai N, Buring JE, et al. 2003. Blood pressure, C-reactive protein, and risk of future cardiovascular events. Circulation, 108:2993-9.

Bots ML, Hoes AW, Koudstaal PJ, et al. 1997. Common carotid intimamedia thickness and risk of stroke and myocardial infarction: the Rotterdam study. Circulation, 96:1432-7.

Casale PN, Devereux RB, Milner M, et al. 1986. Value of echocardiographic measurement of left ventricular mass in predicting cardiovascular morbid events in hypertensive men. Ann Intern Med, 105:173-8.
Chobanian AV, Bakris GL, Black HR, et al. 2003. The Seventh Report of the Joint National Committee on Prevention, Detection, Evaluation, and Treatment of High Blood Pressure: the JNC 7 report. JAMA, 289:2560-72.

Cuspidi C, Meani S, Fusi V, et al. 2004. Metabolic syndrome and target organ damage in untreated essential hypertensives. J Hypertens, 22:1991-8.

Dahlöf B, Devereux RB, Kjeldsen SE, et al. 2002. Cardiovascular morbidity and mortality in the Losartan Intervention For Endpoint reduction in hypertension study (LIFE): a randomised trial against atenolol. Lancet, 359: 995-1003.

Dahlöf B, Sever PS, Poulter NR, et al. 2005. Prevention of cardiovascular events with an antihypertensive regimen of amlodipine adding perindopril as required versus atenolol adding bendroflumethiazide as required, in the Anglo-Scandinavian Cardiac Outcomes Trial-Blood Pressure Lowering Arm (ASCOT-BPLA): a multicentric randomised controlled trial. Lancet, 366:895-906.

De Backer G, Ambrosioni E, Broch-Johnsen K. 2003. European guidelines on cardiovascular disease prevention in clinical practice Third Joint Task Force of European and other Societies on Cardiovascular Disease Prevention in Clinical Practice. Eur J Cardiovasc Prev Rehab, 10(Suppl 1): S1-78.

Devereux RB, Alderman MH. 1993. Role of preclinical cardiovascular disease in the evolution from risk factor exposure to development of morbid events. Circulation, 88:1444-55.

Devereux RB, Dahlöf B, Gerdts E, et al. 2004. Regression of left ventricular hypertrophy by losartan compared with atenolol. The Losartan Intervention For Endpoint reduction in hypertension (LIFE) trial. Circulation, 110:1456-62.

Guidelines Subcommittee. 2003. European Society of Hypertension-European Society of Cardiology Guidelines for the management of arterial hypertension. J Hypertens, 21:1011-53.

Grundy SM. 1999. Hypertriglyceridemia, insulin resistance, and the metabolic syndrome. Am J Cardiol, 83:25F-9.

Hansson L, Zanchetti A, Carruthers SG, et al. 1998. Effects of intensive blood-pressure lowering and low-dose aspirin in patients with hypertension: principal results of the Hypertension Optimal Treatment (HOT) randomised trial. HOT Study Group. Lancet, 351:1755-62.

Isles CG, Walker LM, Beevers GD, et al. 1986. Mortality in patients of the Glasgow Blood Pressure Clinic. J Hypertens, 4:141-56.

Jensen JS, Feldt-Rasmussen B, Strandgaard S, et al. 2000. Arterial hypertension, microalbuminuria, and risk of ischemic heart disease. Hypertension, 35:898-903.

Julius S, Kjeldsen SE, Weber M, et al. 2004. Outcomes in hypertensive patients at high cardiovascular risk treated with regimens based on valsartan or amlodipine: the VALUE randomised trial. Lancet, 363:2022-31.

Klingbeil AU, Schneider M, Martus P, et al. 2003. A meta-analysis of the effects of treatment on left ventricular mass in essential hypertension. Am J Med, 115:41-6.

Koren MJ, Devereux RB, Casale PN, et al. 1991. Relation of left ventricular mass and geometry to morbidity and mortality in uncomplicated essential hypertension. Ann Intern Med, 114:345-52.

Mancini J, Dahlöf B, Díez J. 2004. Surrogate markers for cardiovascular disease. Structural markers. Circulation, 109(Suppl IV):IV-22-30.

Mule G, Cottone S, Nardi E, et al. 2006. Metabolic syndrome in subjects with essential hypertension: relationsship with subclinical cardiovascular and renal damage. Minerva Cardioangiol, 54:173-94.

Nakao N, Yoshimiura A, Morita H, et al. 2003. Combination treatment of angiotensin-II receptor blocker and angiotensin-converting-enzyme inhibitor in non-diabetic renal disease (COOPERATE): a randomised controlled trial. Lancet, 361:117-24.

Nissen SE, Murat E, Libby P, et al. 2004. Effect of antihypertensive agents on cardiovascular events in patients with coronary disease and normal blood pressure. The CAMELOT Study: A randomized controlled trial. JAMA, 292:2217-26. 
Parving HH, Lehnert H, Brochner-Mortensen J, et al. 2001. The effect of irbesartan on the development of diabetic nephropathy in patients with type 2 diabetes. $N$ Engl J Med, 345:870-8.

Pontremolli R, Ravera M, Bezante GP, et al. 1999. Left ventricular geometry and function in patients with essential hypertension and microalbuminuria. J Hypertens, 17:993-1000.

Pontremolli R, Leoncini G, Ravera M, et al. 2002. Microalbuminuria, cardiovascular, and renal risk in primary hypertension. J Am Soc Nephrol, 13:S169-72.

Reboldi G, Gentile G, Angeli, et al. 2005. Microalbuminuria and hypertension. Minerva Med, 96:261-75.

Ridker PM, Rifai N, Rose L, et al. 2002. Comparison of C-reactive protein and low-density lipoprotein cholesterol levels in the prediction of first cardiovascular events. N Engl J Med, 347:1557-65.

Ridker PM. 2003. Clinical application of C-reactive protein for cardiovascular disease detection and prevention. Circulation, 107:363-9.

Ruggenenti P, Fassi A, Ilieva AP, et al. 2004. Preventing microalbuminuria in type 2 diabetes. $N$ Engl J Med, 351:1941-51.

Schillaci G, Pirro M, Vaudo G, et al. 2004. Prognostic values of the metabolic syndrome in essential hypertension. J Am Coll Cardiol, 43:1817-22.

Schrier RW, Estacio RO, Esler A, et al. 2002. Effects of aggressive blood pressure control in normotensive type 2 diabetic patients on albuminuria, retinopathy and strokes. Kidney Int, 61:1086-97.

Shigematsu Y, Hamada M, Ohtsuka T, et al. 1998. Left ventricular geometry as an independent predictor for extracardiac target organ damage in essential hypertension. Am J Hypertens, 11:1171-7.

Sierra C, de la Sierra A, Paré JC, et al. 2002. Correlation between silent cerebral white matter lesions and left ventricular mass and geometry in essential hypertension. Am J Hypertens, 15: 507-12.
Simon A, Gariépy J, Moyse D, et al. 2001. Differential effects of nifedipine and co-amilozide on the progression of early carotid wall changes. Circulation, 103: 2949-54.

UK Prospective Diabetes Study Group. 1998. Efficacy of atenolol and captopril in reducing risk of macrovascular and microvascular complications in type 2 diabetes: UKPDS 39. BMJ, 317:713-20.

Vasan RS, Larson MG, Leip EP, et al. 2001. Impact of high-normal blood pressure on the risk of cardiovascular disease. $N$ Engl J Med, 345:1291-7.

Verdecchia P, Schillaci G, Borgioni C, et al. 1998. Prognostic significance of serial changes in left ventricular mass in essential hypertension. Circulation, 97:48-54.

Viberti G, Wheeldon NM. 2002. Microalbuminuria reduction with valsartan in patients with type 2 diabetes mellitus. A blood pressure-independent effect. Circulation, 106: 672-8.

Weber MA. Julius S, Kjeldsen SE, et al. 2004. Blood pressure dependent and independent effects of antihypertensive treatment on clinical events in the VALUE trial. Lancet, 363: 2049-51.

Wilson AM, Ryan MC, Boyle AJ. 2006. The novel role of C-reactive protein in cardiovascular disease: risk marker or pathogen. Int J Cardiol, 106:291-7.

Zanchetti A, Bond G, Hennig M, et al. 2002. Calcium antagonist lacidipine slows down progression of asymptomatic carotid atherosclerosis. Principal results of the European Lacidipine Study on Atherosclerosis (ELSA), a randomized, double blind, long-term trial. Circulation, 106:2422-7. 